\title{
Four Cases of Hemangiopericytoma and the Contribution of Radiotherapy in Their Management
}

\author{
Bhargavi llangovan ${ }^{1}$ Neetu Sasikumar ${ }^{1}$ Janos Stumpf ${ }^{1}$ Rathna Devi ${ }^{1}$ Murli Venkatraman ${ }^{2}$ \\ S. Kalyanaraman ${ }^{3}$ Chendhilnathan ${ }^{3}$ \\ ${ }^{1}$ Department of Radiation Oncology, Apollo Speciality Hospitals, \\ Chennai, Tamil Nadu, India \\ ${ }^{2}$ Department of Medical Physics, Apollo Speciality Hospitals, \\ Chennai, Tamil Nadu, India \\ ${ }^{3}$ Department of Neurosurgery, Apollo Speciality Hospitals, \\ Chennai, Tamil Nadu, India \\ Indian J Neurosurg 2017;6:20-26.

\begin{abstract}
Address for correspondence Dr. Bhargavi llangovan, MBBS, DMRT Department of Radiation Oncology, Apollo Cancer Hospitals, 320, PADMA Complex, Cenotaph Road, Teynampet, Chennai-35, Tamil Nadu, India (e-mail: bhargavi.ilangovan@gmail.com).
\end{abstract}

\begin{abstract}
Hemangiopericytomas are tumors arising from the Zimmerman pericytes. They are characterized by their propensity to recur aggressively and metastasize. Surgery forms the primary treatment modality. Radiotherapy has been used in the postoperative setting and has proved to produce a significant progression-free survival in cases with residual disease. Radiotherapy has been applied even as a single modality treatment whenever surgery could not be performed. We are presenting four cases of meningeal hemangiopericytomas which have been treated with radiotherapy in combination with surgery. These cases show various scenarios in which Radiotherapy has been used in

Keywords

- hemangiopericytoma

- radiotherapy

- radiosurgery hemangiopericytoma: adjuvant setting, residual/recurrent setting, and in the palliative setting. As far as the technique is concerned, three-dimensional conformal radiotherapy, fractionated radiosurgery, and other forms of high-precision irradiation (intensity-modulated radiation therapy [IMRT]) were given.
\end{abstract}

\section{Introduction}

Hemangiopericytomas (HPC) are neoplasms arising from the Zimmermann pericytes surrounding the capillaries. These are contractile cells with long processes which wrap themselves around capillaries and serve to change the caliber of their lumens. Zimmermann et al have suggested that these pericytes are modified smooth muscle cells. ${ }^{1}$ These tumors tend to be well circumscribed and consist of tightly packed cells around thin-walled vascular channels of varying calibers. There is a slight male predilection for the tumor. About $90 \%$ of the tumors arise in persons between the age of 50 and 70 years. No specific etiologic factor is known. They develop mainly in the lower extremities, retro peritoneum, or pelvis in adults. Approximately, 15 to $30 \%$ occur in the head and neck. ${ }^{2}$

received

April 6, 2015

accepted

May 25, 2015

published online

July 13, 2016
DOI http://dx.doi.org/

$10.1055 / \mathrm{s}-0036-1582433$ ISSN 2277-954X.
In the central nervous system (CNS), they are mostly dural based and display locally aggressive behavior but may metastasize too. On the basis of MENA's classification, ${ }^{3}$ the original tumors were divided into differentiated and anaplastic. Anaplastic HPC are characterized by the presence of necrosis and/or greater than five mitoses per ten microscopic fields (magnification $\times 400$ ), and at least two of the following microscopic features: hemorrhage, moderate-to-high nuclear atypia, and moderate-to-high cellularity. Surgery forms the mainstay of treatment for the tumor, ${ }^{4}$ but radiation too is given in a majority of the cases.

Our four cases give us the opportunity to report on the extremely wide range of efficacy radiation can be considered. Reirradiation of the same area was done in three cases.
License terms

$(\circledast) \Theta \circledast$ (c) 2017 Neurological Surgeons' Society
of India

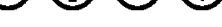




\section{Case Reports}

\section{Case 1}

A 68-year-old man was evaluated for intermittent giddiness of 3 months duration in November 2007. Magnetic resonance imaging (MRI) of the brain showed a destructive lesion involving occipital bone with intracranial and extra cranial extension. He underwent posterior fossa craniotomy and subtotal excision of the tumor in December 2007. Histopathology revealed a low grade HPC. He received (postoperative) radiotherapy of $60 \mathrm{~Gy}$ in 30 fractions to the residual tumor from December 17, 2007, to February 1, 2008 , by three-dimensional conformal radiotherapy (3DCRT) technique. The patient was on regular follow-up. He was asymptomatic for 30 months. He presented with unsteady gait and back pain in October 2010. He was found to have a spinal lesion in the fifth dorsal vertebra with soft tissue component (-Fig. 1a).

He underwent a decompressive surgery and the histopathology was consistent with HPC. He received radiotherapy with a total dose of $57 \mathrm{~Gy}$ (27 Gy in 15 fractions to D4 to D7 vertebrae encompassing D5 and the soft tissue component by 3DCRT technique and a boost of $30 \mathrm{~Gy}$ in 15 fractions to the D5 vertebra) by high-precision irradiation (IMRT) technique from November 3, 2010, to December 24, 2010 (-Fig. 1b).

Follow-up MRI in February 2011 showed an interval increase in the metastatic involvement in the transverse process of D5, an expansile lesion in the left iliac bone and bilateral femora. Bone scan also showed an increased
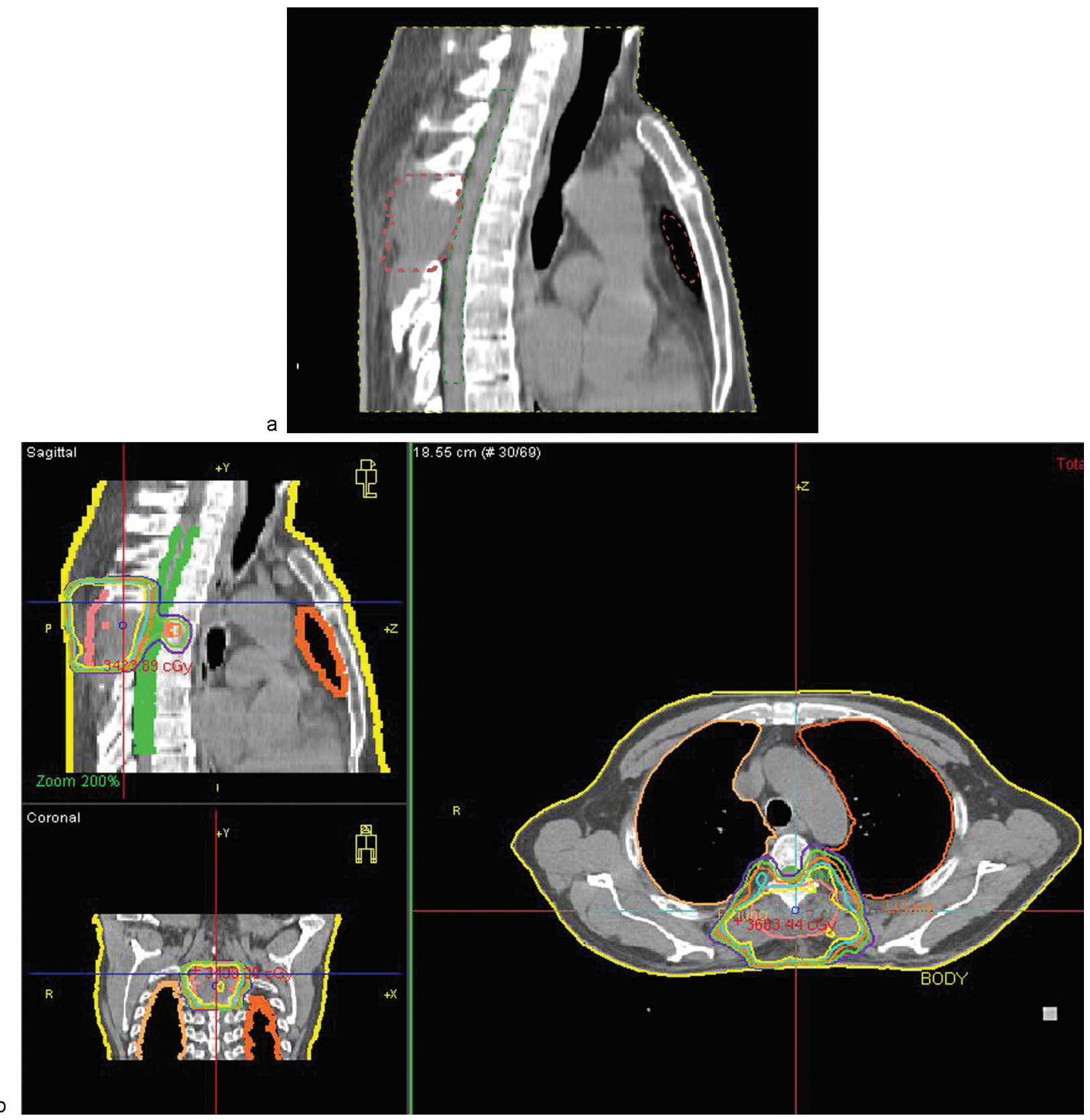

Fig. 1 (a) The spinal lesion with soft tissue component after surgery. (b) Intensity-modulated radiation therapy plan for the D5 vertebra. 
tracer uptake in the aforementioned areas and the left scapula. Positron emission tomography (PET) scan showed a residual lesion in the posterior fossa with no significant fluorodeoxyglucose (FDG) uptake. He received palliative radiotherapy to the bilateral femora, left scapula (30 Gy in 10 fractions) from February 16, 2011, to March 15, 2011. In December 2011, follow-up PET scan showed multiple bone metastases with soft tissue component and cortical breach in the right iliac bone and left scapula with a stable primary. He received radiotherapy of $50 \mathrm{~Gy}$ in 25 fractions to the bilateral femora and the right iliac bone from December 22, 2011, to January 25, 2012, by 3DCRT technique. In March 2012, he was evaluated for projectile vomiting and was found to have a marginal increase in the size of the primary tumor in the posterior fossa compressing the fourth ventricle and the brain stem. He underwent ventriculoperitoneal shunt placement on February 25, 2012. He was treated with stereotactic radiosurgery using Cyber knife with a total dose of $25 \mathrm{~Gy}$ (-Fig. 2) in five fractions from April 2, 2012, to April 7, 2012 , prescribed to the $87 \%$ isodose line. He was marginally symptomatic 2 months later and died at home after approximately 7 months (details unknown).

\section{Case 2}

A 26-year-old gentleman presented with the complaints of giddiness and vomiting in March 2003. On evaluation, he was found to have a posterior fossa space occupying lesion for which he underwent a craniotomy and complete tumor excision. Histopathology revealed an HPC. He was asymptomatic for 33 months. In February 2006, he presented with headache and was found to have a recurrence in the left cerebellopontine angle. He underwent a reexploration and partial tumor excision followed by postoperative radiotherapy of $60 \mathrm{~Gy}$ in 30 fractions by IMRT technique by June 2006 . He had a symptom-free interval of 26 months. In August 2008, he developed giddiness and difficulty in walking and was

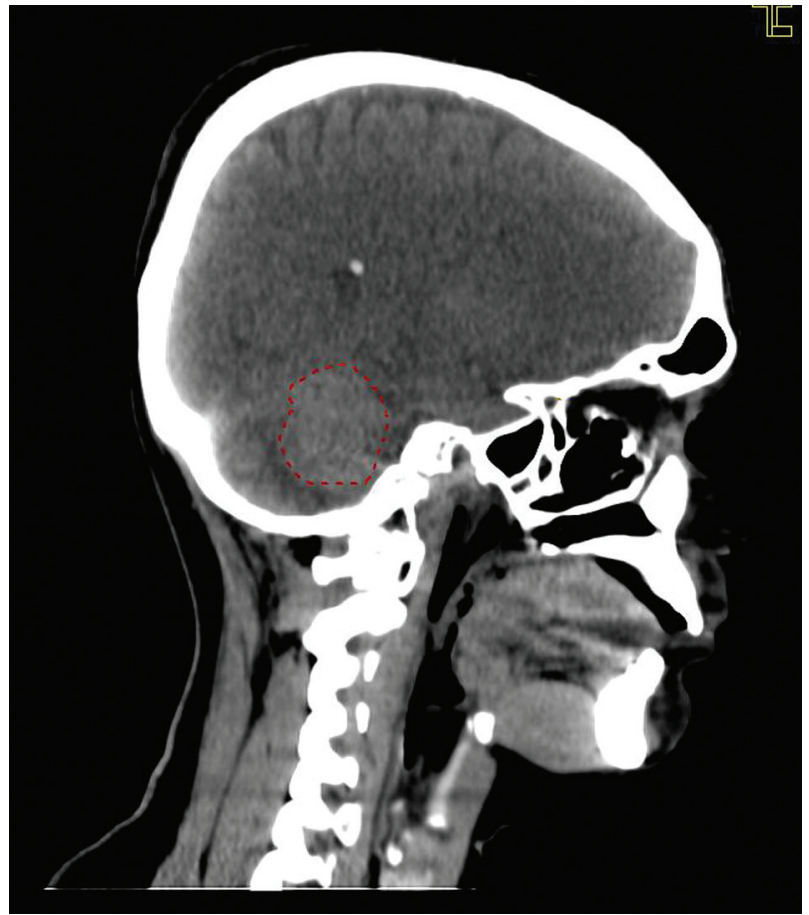

Fig. 3 The target volume of his second irradiation.

diagnosed with local recurrence. He underwent retro mastoid craniotomy and near total excision of the tumor followed by postoperative radiotherapy with a dose of $55.8 \mathrm{~Gy}$ in 31 fractions by IMRT technique from September 9, 2008, to November 9, 2008 (-Fig. 3).

During the radiotherapy treatment, patient had a ventriculoperitoneal shunt (October 14, 2008). In August 2009, he had complaints of diplopia and left facial nerve palsy. On evaluation, he was found to have a recurrent lesion in the posterior fossa. He underwent neuronavigationguided left petrosectomy and microscopic near total excision of the lesion on September 21, 2009. HPE and IHC confirmed HPC.

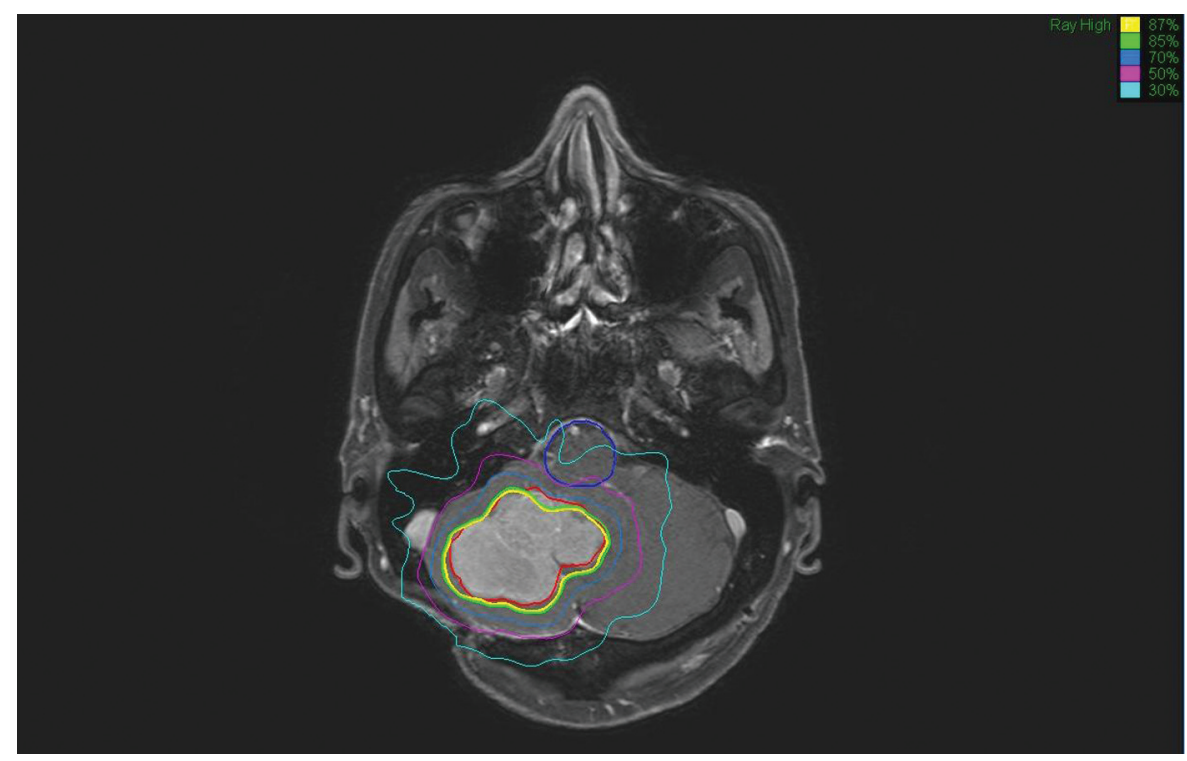

Fig. 2 Magnetic resonance imaging showing the target and the dose at the time of the second irradiation. 
Follow-up scan revealed a $1.5-\mathrm{cm}$ residual lesion, close to the surgical area. Neither the neurosurgeon nor the patient was interested in surgery. He was taken up for fractionated stereotactic radio surgery. A dose of $30 \mathrm{~Gy}$ in four fractions was delivered using Cyber knife from February 8, 2011, to February 11, 2011 (-Fig. 4). Patient was asymptomatic after third irradiation for approximately 18 months and again became symptomatic with progressive disease.

\section{Case 3}

A 46-year-old gentleman was evaluated for the complaints of gait disturbance and headache in November 2004 and was found to have a posterior fossa tumor. He underwent suboccipital craniectomy and excision of the tumor which was reported as a cerebellar HPC. He was asymptomatic for 24 months and had a local recurrence in December 2006 for which he underwent suboccipital craniotomy and subtotal excision. He also received postoperative radiotherapy to the residual tumor with a dose of $60 \mathrm{~Gy}$ in 30 fractions by IMRT technique from January 9, 2007, to February 21, 2007. He was on periodic follow-up and interim analysis showed a decrease in the tumor volume. In April 2012, he noted an occipital swelling with headache. His MRI showed a local recurrence at the edge of the originally irradiated area. Neurosurgical opinion was sought and there was a major reluctance to do an open surgery because of the serious risks involved. He received fractionated stereotactic radio surgery using CyberKnife (Accuray, Sunnyvale, California, United States) with a dose of $35 \mathrm{~Gy}$ in five fractions prescribed to the $90 \%$ isodose line which was encompassing the visible tumor bulk only from May 23, 2012, to May 28, 2012 (-Fig. 5). He tolerated the treatment well. He is on follow-up and showed improvement 4 months after radiosurgery.

\section{Case 4}

A 59-year-old male patient was treated for a skull base tumor with palatotomy in 2001. Histopathology was reported as HPC. In December 2006, he had complaints of nasal block and difficulty in breathing. MRI of the brain revealed a large lobulated lesion in the right infratemporal fossa and parapharyngeal space reaching the skull base. He underwent mandibulectomy and excision of the right parapharyngeal and infratemporal fossa mass. Histopathology was consistent with HPC. He was on regular follow-up. Follow-up computed tomographic scan showed enhancing nodular mass lesion involving the nasopharynx

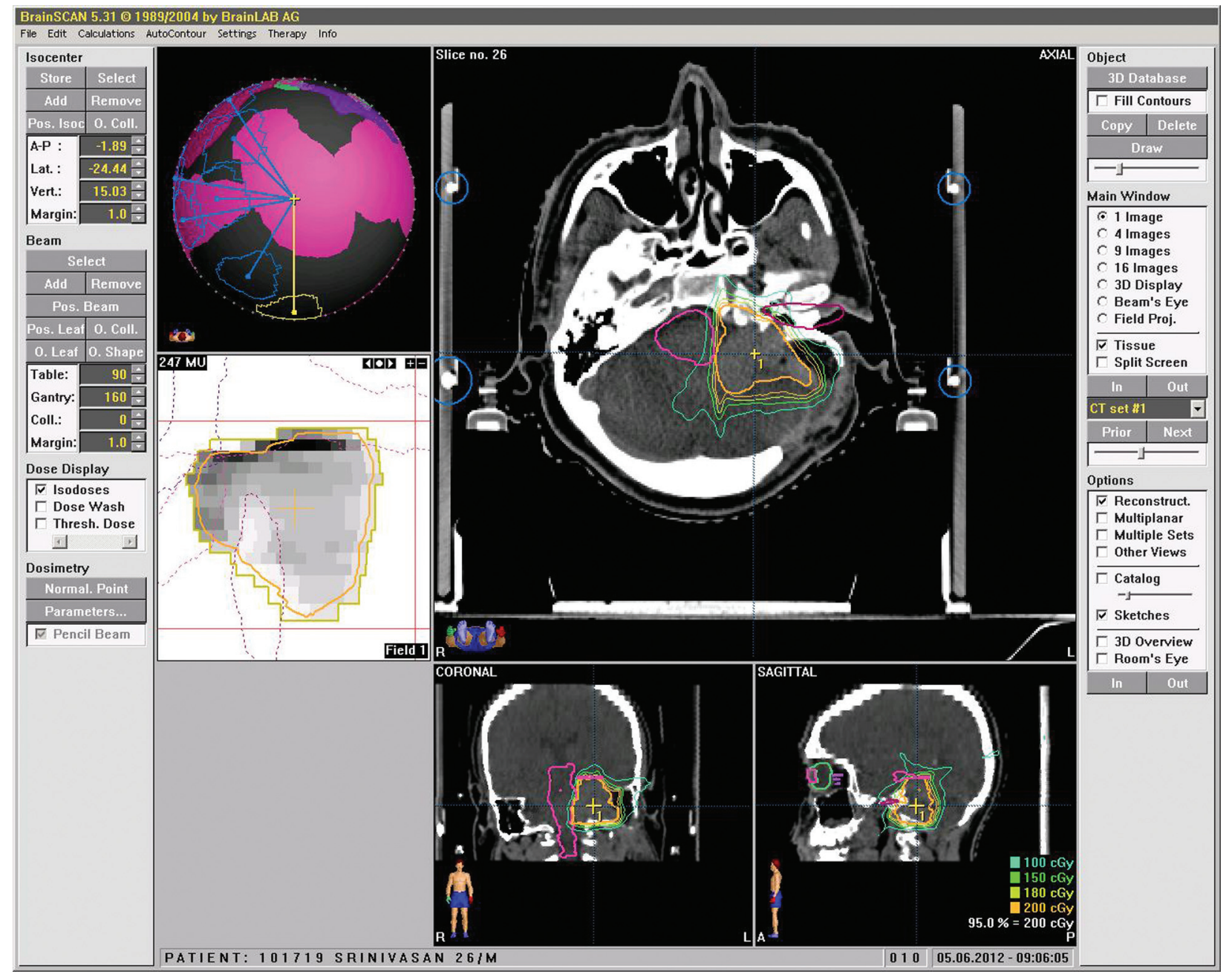

Fig. 4 Target volume and isodose lines during the second irradiation of the primary. 


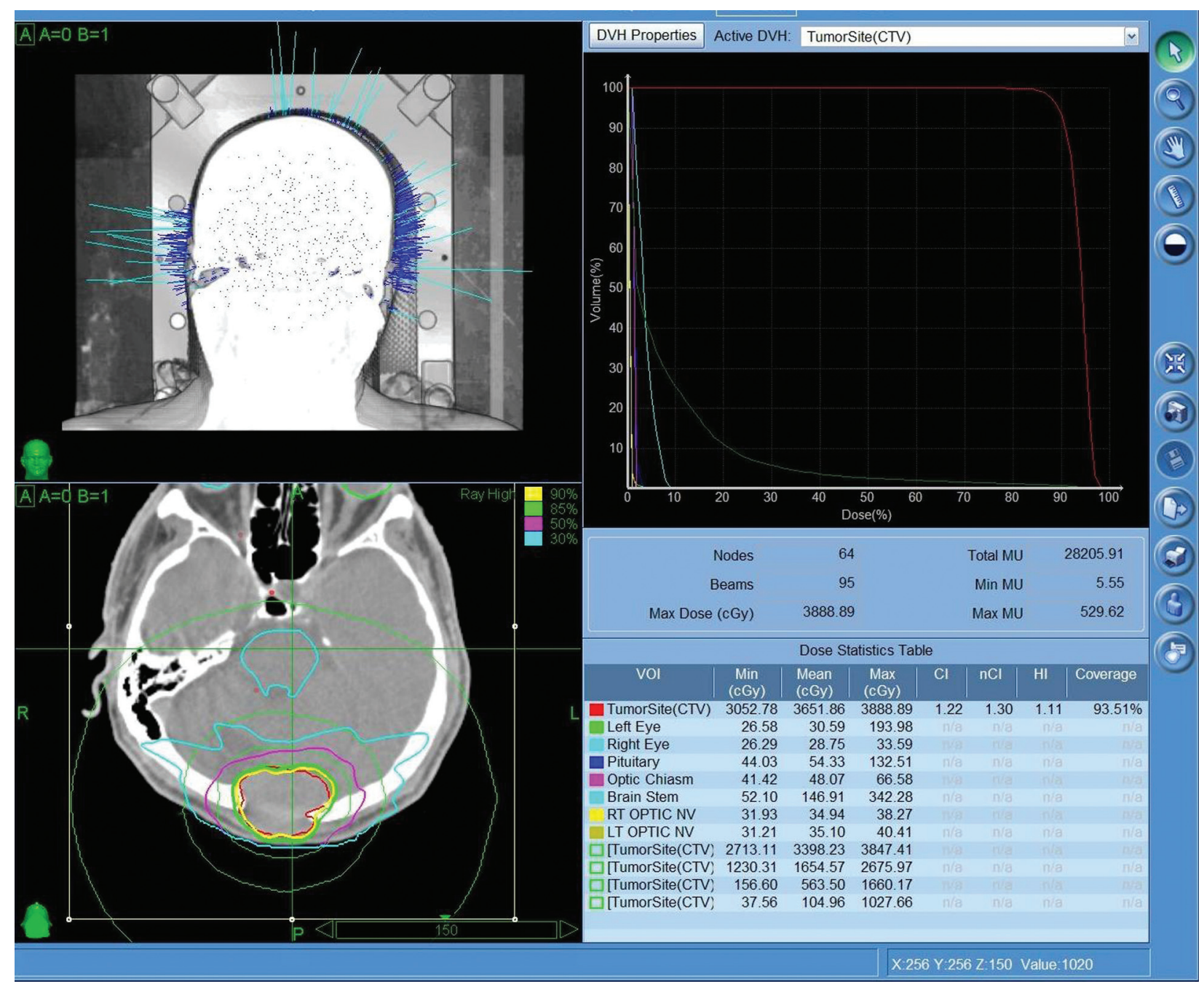

Fig. 5 Treatment plan.

and right tonsillar fossa with postoperative changes in the right submandibular region. He was asymptomatic for a period of 54 months. He presented in May 2012 with change in voice, difficulty in swallowing, and headache. He was put on Ryle tube due to regurgitation and risk of aspiration. MRI showed tumor in the Clivus and the jugular foramen area. As surgery could lead to permanent loss of hearing on the side and could also cause irreversible injury to the lower cranial nerves, he was not operated upon. He was referred for radiotherapy. He was started with hypofractionated radiotherapy with CyberKnife (-Fig. 6). After he had significant pain relief, it was decided to treat him with conventionally fractionated radiotherapy. He received a dose of $21 \mathrm{~Gy}$ in three fractions using CyberKnife radiosurgery from May 21, 2012, to May 25, 2012, followed by a dose of $50.4 \mathrm{~Gy}$ in 28 fractions using IMRT technique from May 31, 2012, to July 7, 2012. Patient had significant symptomatic relief at the end of the treatment. Now after 5 months of treatment, patient has good pain relief and his regurgitation is practically gone. He is without Ryle tube and has gained weight. The volume analysis shows good regression of tumor (from 37,764.41 to 6,123.54 mL) (-Fig. 7).

\section{Discussion}

HPCs are tumors of Zimmerman pericytes. They are characterized by their propensity to recur aggressively and metastasize. Meningeal HPCs are rare vascular tumors which are most commonly diagnosed in the early fifth decade of life and accounts for $1 \%$ of all CNS tumors. Although initially it was believed to a meningioma variant (angioblastic meningioma), they have been recognized as a distinct pathologic entity with different clinical behavior, immunohistochemical characteristics, and ultrastructural features compared with meningiomas. ${ }^{5}$ They are primarily treated with surgery. A complete tumor excision or good surgical margins is not always possible because of the position, local adherence, and invasion.

External beam radiotherapy has been used with different intentions as adjuvant therapy to surgery for the treatment of primary and local recurrences, following complete surgical resection, with curative intention or as a repeated radiotherapy attempt to control disease. At a focal conventionally fractionated dose of more than $50 \mathrm{~Gy}$, studies have shown a significant increase in the length of time to tumor progression. ${ }^{6}$ 


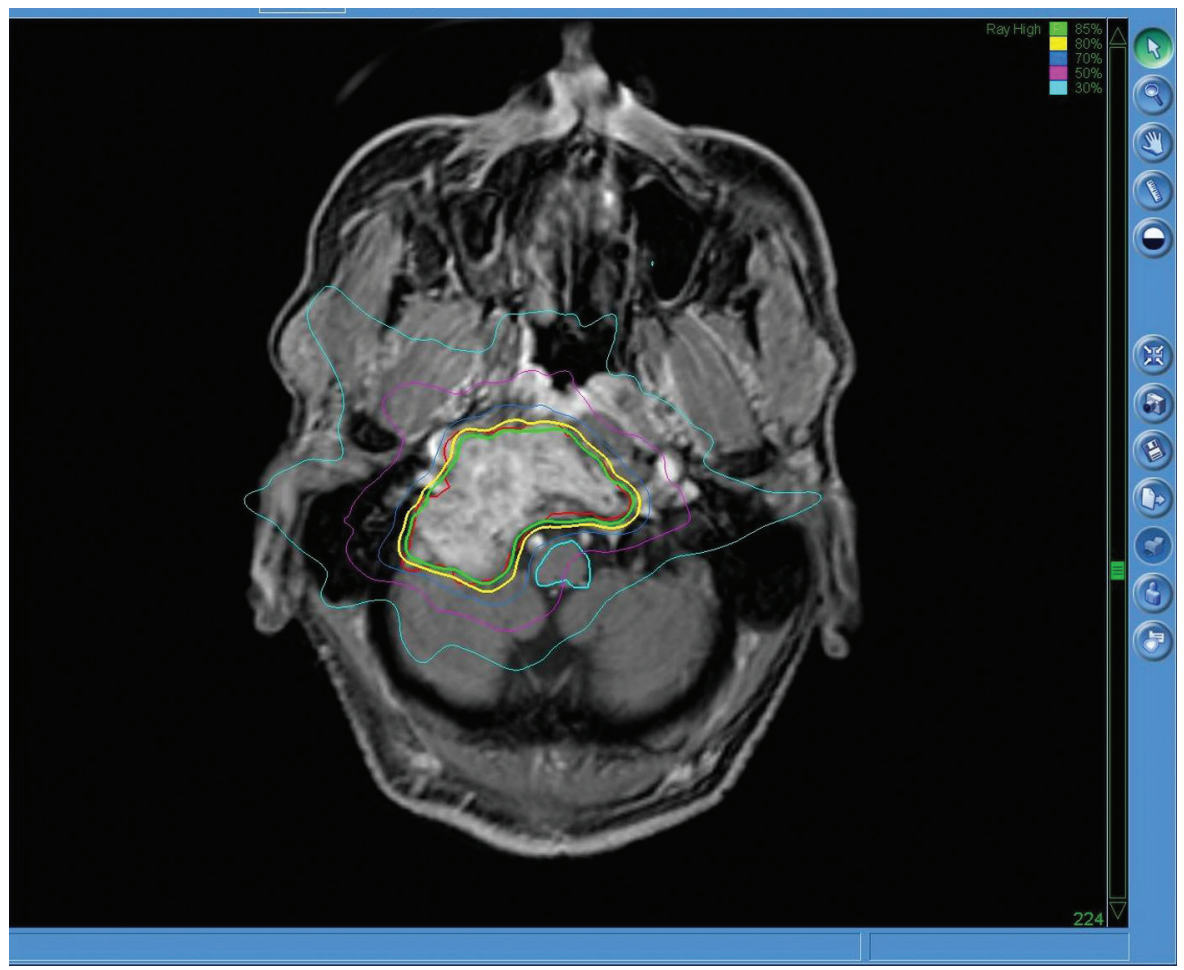

Fig. 6 Radiosurgery treatment plan.

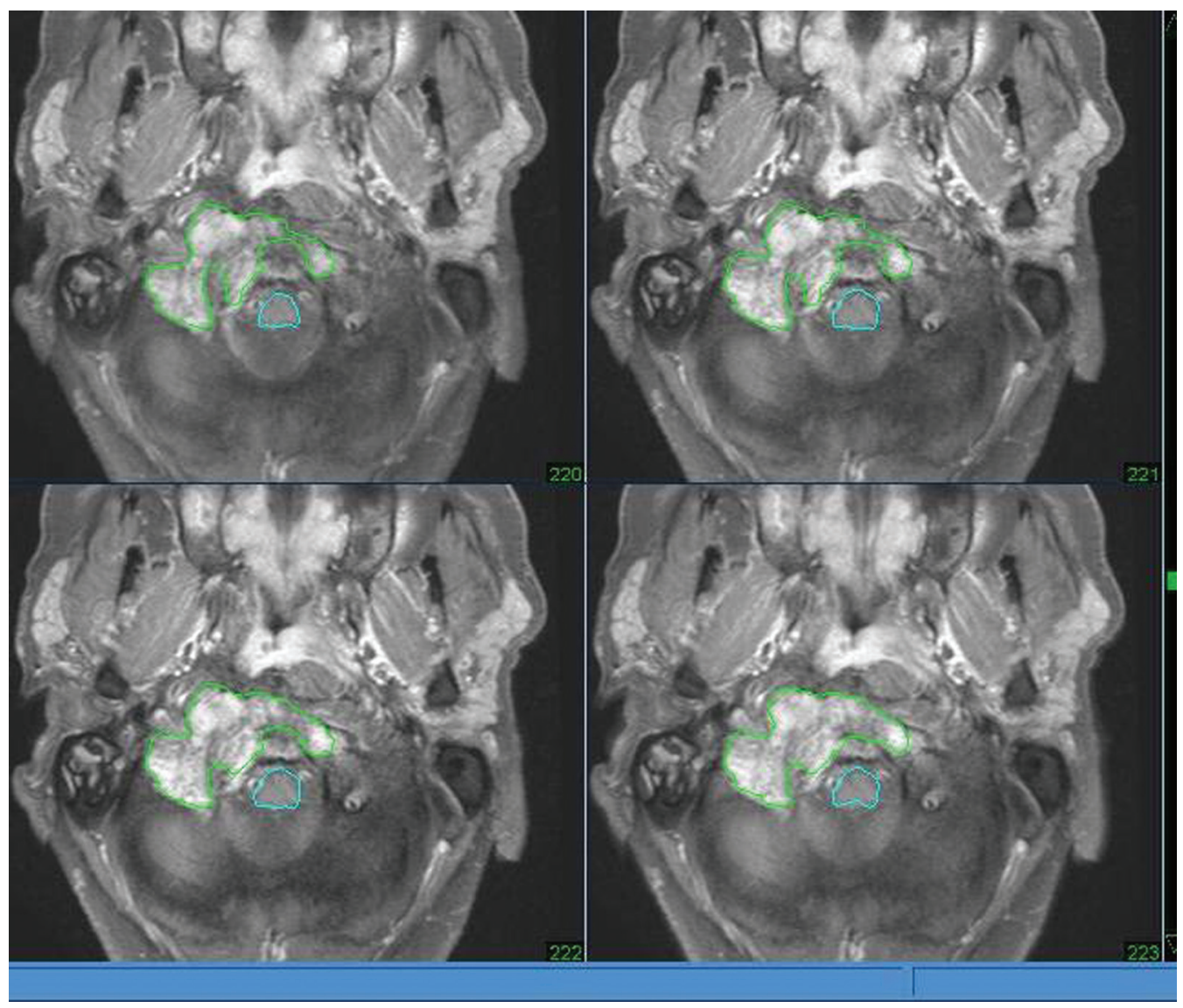

Fig. 7 Posttreatment volume in the magnetic resonance imaging. 
The role of radiotherapy is controversial indeed. It is quite clear that it can control the tumor. But the dose and fractionation of radiotherapy that can be curative has to be understood. The presence of residual tumor after open surgery is a definite indication for radiotherapy as it delays progression. ${ }^{7}$ Radiotherapy is being used in the adjuvant setting after complete removal though literature is controversial. For us, it makes sense to do adjuvant radiotherapy as these tumors are hardly ever removed with a good surgical margin. The ideal dose in conventional fractionation is not clear either. In our cases, we felt to prescribe the maximum dose tolerable to the environment. The use of nonconventional irradiation like radiosurgery and fractionated radiosurgery is not yet evolved. Reirradiation for local recurrence with minimal side effects and prolonged survival is very likely with radiosurgery. ${ }^{8}$

Our four cases of HPCs were primarily treated with surgery. Radiotherapy had been used in all the cases, but differently. It has been given in the postoperative setting, where it has delayed the progression of the residual disease as in case 1 . In local recurrences, radiotherapy has been used to arrest the further growth of the tumor and to cause symptomatic relief as in case 2 . It has also been used to palliate distant metastases in one of our patients (case 1) and has produced the desired effect. Reirradiation in the same area was delivered using fractionated radiosurgery with CyberKnife, thereby minimizing exposure of surrounding tissues. HPCs are slow growing tumors and hence radiosurgery has a role in their management. In our cases radiosurgery was used as in combination with IMRT for one case, as palliation for recurrent HPCs 6 years after surgery. One patient was irradiated in the infratentorial area, thrice and IMRT twice, radiosurgery (case 2).

\section{Conclusion}

Radiotherapy has a definite role in the treatment of HPCs. It is supposed to increase the progression free survival of patients thereby improving survival. ${ }^{9}$ It is also effective in palliating distant metastases. Radiosurgery has theoretically added advantage because of the ability to deliver large doses with marginal sparing of normal tissue. HPCs were treated with a large fraction doses in case of reirradiation. Patients have shown good clinical response to irradiation and to repeated radiation. One case had a third irradiation with fractionated radiosurgery close to the brain stem. It has resulted in response both clinically and image documented with no obvious side effect. This too clearly indicates that, high precision radiotherapy and even radiosurgery are having an increasing role in the complex management of this pathology. In general, we may conclude that highprecision irradiation and maximum sparing of sensitive tissues is warranted, as this tumor is recurring and the repeated irradiation to the same area remains a reasonable treatment option to improve the quality of life, to shrink the tumor mass, and to prolong survival.

\section{References}

1 Stout AP, Murray MR. Hemagiopericytoma: vascular tumor featuring Zimmerman's pericytes. Ann Surg 1942;116(1): 26-33

2 Enzinger FM, Smith BH. Hemangiopericytoma. An analysis of 106 cases. Hum Pathol 1976;7(1):61-82

3 Mena H, Ribas JL, Pezeshkpour GH, Cowan DN, Parisi JE. Hemangiopericytoma of the central nervous system: a review of 94 cases. Hum Pathol 1991;22(1):84-91

4 Lamar Z, Lesser GJ. Management of meningeal neoplasms: meningiomas and hemangiopericytomas. Curr Treat Options Oncol 2011;12(3):230-239

5 Nunnery EW, Kahn LB, Reddick RL, Lipper S. Hemangiopericytoma: a light microscopic and ultrastructural study. Cancer 1981;47(5): 906-914

6 Staples JJ, Robinson RA, Wen BC, Hussey DH. Hemangiopericytomathe role of radiotherapy. Int J Radiat Oncol Biol Phys 1990;19(2): 445-451

7 Mira JG, Chu FC, Fortner JG. The role of radiotherapy in the management of malignant hemangiopericytoma: report of eleven new cases and review of the literature. Cancer 1977; 39(3):1254-1259

8 Coffey RJ, Cascino TL, Shaw EG. Radiosurgical treatment of recurrent hemangiopericytomas of the meninges: preliminary results. J Neurosurg 1993;78(6):903-908

9 Guthrie BL, Ebersold MJ, Scheithauer BW, Shaw EG. Meningeal hemangiopericytoma: histopathological features, treatment, and long-term follow-up of 44 cases. Neurosurgery 1989;25(4): 514-522 